This item was submitted to Loughborough's Research Repository by the author.

Items in Figshare are protected by copyright, with all rights reserved, unless otherwise indicated.

\title{
Time-dependent rheological behaviour of bacterial cellulose hydrogel
}

\section{PLEASE CITE THE PUBLISHED VERSION}

http://dx.doi.org/10.1016/j.msec.2015.08.019

\section{PUBLISHER}

(c) 2015 Elsevier B.V.

\section{VERSION}

AM (Accepted Manuscript)

\section{PUBLISHER STATEMENT}

This work is made available according to the conditions of the Creative Commons Attribution-NonCommercialNoDerivatives 4.0 International (CC BY-NC-ND 4.0) licence. Full details of this licence are available at: https://creativecommons.org/licenses/by-nc-nd/4.0/

\section{LICENCE}

CC BY-NC-ND 4.0

\section{REPOSITORY RECORD}

Gao, Xing, Zhijun Shi, Piotr Kusmierczyk, Changqing Liu, Guang Yang, Igor Sevostianov, and Vadim V. Silberschmidt. 2019. "Time-dependent Rheological Behaviour of Bacterial Cellulose Hydrogel". figshare. https://hdl.handle.net/2134/19472. 


\title{
TIME-DEPENDENT RHEOLOGICAL BEHAVIOUR OF BACTERIAL CELLULOSE HYDROGEL
}

\author{
Xing Gao ${ }^{1}$, Zhijun Shi ${ }^{2}$, Piotr Kuśmierczyk ${ }^{3}$, Changqing Liu ${ }^{1}$, Guang Yang ${ }^{2}$, Igor \\ Sevostianov ${ }^{4}$ and Vadim V Silberschmidt ${ }^{1}$ \\ ${ }^{1}$ Wolfson School of Mechanical and Manufacturing Engineering, Loughborough University, \\ Loughborough, UK. \\ ${ }^{2}$ College of Life Science and Technology, Huazhong University of Science and Technology, \\ Wuhan, China. \\ ${ }^{3}$ Department of Mathematics, Aberystwyth University, Aberystwyth, UK. \\ ${ }^{4}$ Department of Mechanical and Aerospace Engineering, New Mexico State University, Las \\ Cruces, USA.
}

\begin{abstract}
Present work focuses on time-dependent rheological behaviour of bacterial cellulose (BC) hydrogel. Due to its ideal biocompatibility, BC hydrogel could be employed in biomedical applications. Considering the complexity of loading conditions in human body environment, time-dependent behaviour under relevant conditions should be understood. BC specimens are produced by Gluconacetobacter xylinus ATCC 53582 at static-culture conditions. Time-dependent behaviour of specimens at several stress levels is experimentally determined by uniaxial tensile creep tests. We use fraction-exponential operators to model the rheological behaviour. Such a representation allows combination of good accuracy in analytical description of viscoelastic behaviour of real materials and simplicity in solving boundary value problems. The obtained material parameters allow us to identify time-dependent behaviour of BC hydrogel at high stress level with sufficient accuracy.
\end{abstract}

Key words: bacterial cellulose hydrogel; rheology; creep test; fraction-exponential operators; time-dependent behaviour

\section{Introduction}

Bacterial cellulose (BC) hydrogel (FIG. 1a) is known for its excellent biocompatibility (Shi et al., 2014). It makes BC hydrogels a potential material for various bioengineering applications, such as implant replacement of human tissues (Malm et al., 2012; Wang et al., 2010; Zang et al., 2015), encasement for tissue regeneration (Bäckdahl et al., 2006; Bäckdahl et al., 2008; Gibas 
and Janik, 2010; Kowalska-Ludwicka et al., 2013), etc. From a structural point of view, BC hydrogel is a typical nonwoven bio-material. It consists of high-crystalline (70 80\%, Klemm et al., 2006) long nanofibres that are randomly distributed in its radial-transverse plane (FIG. 1b). Fibrous layers with some cross-links form a multi-layer structure in the out-of-plane direction (FIG. 1c). Such a fibrous scaffold with high porosity ( 99 vol\%) is capable to hold a large amount of interstitial water, forming the hydrogel. The growing interest to $\mathrm{BC}$ is not only thanks to its excellent biological properties but also because of attractive mechanical properties of BC nanofibres (Guhados et al., 2005). BC fibre-reinforced bio-composites with optimized mechanical properties have found various applications for artificial tissues, such as a BC/fibrin composite for artificial bloody vessel (Brown et al., 2012).

A matrix of the $\mathrm{BC}$ composite is usually acts as a damper in loading-bearing processes; therefore, the BC hydrogel or the composite based on it demonstrates viscoelastic behaviour. Since BC is exposed to complex loading conditions of human-body environment, it is extremely important to characterize its viscoelastic properties in an explicit analytical form. Brown et al. (2011, 2012) quantified elastic and viscoelastic properties of a BC fibre-reinforced bio-composite with tensile and cyclic creep tests, demonstrating its suitability as a potential implant for artificial blood vessels. Nimeskern et al. (2013) evaluated viscoelastic properties of BCs with various cellulose contents by means of stress-relaxation indentation; then, comparing the obtained data with those for ear cartilage. It was demonstrated that BC could be used as ear-cartilage replacement. Accurate prediction of viscoelastic behaviour using an appropriate theoretical approach is necessary for optimization of mechanical performance of BC under application-relevant conditions. A spring/dashpot combination model is often used to depict creep and stress-relaxation behaviour. Kim et al. (2008) used a model formed by parallel spring-dashpot combinations and a dashpot in series to describe cyclic creep behaviour of cellulose electro-active paper at various temperatures. Frensemeier et al. (2010) used the Burgers viscoelastic model that accounts for water release to decreibe a visocelastic response of a $\mathrm{BC}$ hydrogel. Lopez-Sanchez et al. (2014) studied viscoelastic behaviour of a BC hydrogel with a linear transversely isotropic poroelastic model based on its compression response to loading with various strain rates.

The use of oversimplified spring/dashpot combinations is caused by the fact that a standard approach to solve problems of viscoelasticity is based on elasticity-viscoelasticity correspondence principle (see, for example, Christensen, 1982). The problem is formulated in a Fourier or Laplace domain, treated as the elastic one, and then, an inverse transform gives the desired viscoelastic solution. The main challenge in this approach is obtaining analytical formulas for the inverse transform. It can be achieved only for some particular cases corresponding to various combinations of springs and dashpots. Note, however, that the governing relations of viscoelasticity (as all other relations of this kind) are of a phenomenological nature - they are chosen to match experimental data obtained in standard tests on creep and relaxation. Unfortunately, simplest models describing combinations of springs and 
dashpots are not sufficiently flexible to match experimental data for real materials while more complex ones do not allow analytical expressions for inverse Fourier or Laplace transforms appearing in the solution.

An alternative description of viscoelastic behaviour was proposed by Scott Blair and Coppen $(1939,1943)$ and Rabotnov (1948). They suggested using fraction-exponential operators that, on the one hand, can describe experimental data of real materials with sufficient accuracy and, on the other hand, allow analytical Laplace transformations. Recently, the use of these fraction-exponential operators attracted attention of researchers in the area of solid mechanics (especially mechanics of heterogeneous materials) again; the respective detailed discussion can be found, for instance, in the book of Podlubny (1998). A connection between fraction-exponential operators and various simplistic models involving combinations of dashpots and springs was analysed in the work of Di Paola and Zingales (2012). Applications of fraction-exponential operators to various heterogeneous viscoelastic materials are discussed in Levin and Sevostianov (2005), Sevostianov and Levin (2015) and Sevostianov et al. (2015) . In this paper, a fraction-exponential model is employed to describe a time-dependent behaviour of the BC hydrogel. Tensile creep tests at various stress levels were performed to quantify its mechanical behaviour. A numerical procedure was developed in MATLAB to recover the model parameters from the experimental data.

\section{Fraction exponential operators}

To describe viscoelastic properties of the material, a most general form of the respective governing equation is used in the form of Stieltjes convolution:

$$
\varepsilon_{i j}(x, t)=S_{i j k l} \sigma_{k l}(x, t)+\int_{0}^{t} K_{i j k l}(t-\tau) \sigma_{k l}(x, \tau) d \tau
$$

where $\varepsilon_{i j}$ and $\sigma_{k l}$ are the strain and the stress tensors, respectively, $S_{i j k l}$ is a fourth rank tensor of instantaneous elastic compliance and $K_{i j k l}(t)$ is time-dependent forth-rank tensor (creep kernel) satisfying the fading-memory principle: $K_{i j k l}(t) \underset{t \rightarrow \infty}{\rightarrow 0}$. It is assumed that volume changes during deformation are purely elastic. Then, for an isotropic material, expression (1) can be written as

$$
\varepsilon_{i j}(x, t)=\frac{1}{3 K_{0}}\left(\frac{1}{3} \delta_{i j}\right) \sigma_{k k}(x, t)+\frac{1}{2}\left(\mu^{*}\right)^{-1}\left(\sigma_{i j}(x, t)-\frac{1}{3} \delta_{i j} \sigma_{k k}(x, t)\right)
$$

where 
$\left(\mu^{*}\right)^{-1}[f(x, t)]=\frac{1}{\mu_{0}} f(x, t)+\int_{0}^{t} J(t-\tau) f(x, \tau) d \tau$,

$K_{0}$ is the bulk elastic modulus of the material, $\mu_{0}$ is instantaneous shear modulus, and creep kernel $J(t-\tau)$ satisfies the fading memory principle. To model this kernel, we follow Scott Blair and Coppen (1939, 1943) and Rabotnov (1948) who independently proposed to use fraction-exponential functions for this goal in the form:

$$
J(t-\tau) \equiv \ni_{\alpha}(\beta, t-\tau)=(t-\tau)^{\alpha} \sum_{n=0}^{\infty} \frac{\beta^{n}(t-\tau)^{n(1+\alpha)}}{\Gamma[(n+1)(1+\alpha)]}
$$

As mentioned in the introduction, this representation allows analytical expression for an inverse Laplace transform in solving boundary-value problems, and, at the same time it is sufficiently general to match experimental data with good accuracy. To satisfy the fading memory principle, the following restrictions on the parameters entering (4) have to be satisfied:

$\beta<0 ; \quad-1<\alpha \leq 0$

An operator with such a kernel acts on a unit function as follows:

$\ni_{\alpha}^{*}(\beta, t) \cdot 1=\frac{1}{\beta}\left[\mathrm{M}_{\alpha+1}\left(\beta t^{\alpha+1}\right)-1\right]$

where $\mathrm{M}_{a}(z)$ is the Mittag-Leffler function:

$$
\mathrm{M}_{a}(\mathrm{z})=\sum_{n=0}^{\infty} \frac{\mathrm{z}^{n}}{\Gamma(n a+1)}
$$

The Mittag-Leffler function monotonically decreases from 1 to 0 so that

$$
\lim _{t \rightarrow \infty}\left[\ni_{\alpha}^{*}(\beta, t) \cdot 1\right]=\frac{-1}{\beta}
$$

For an isotropic material, shear operators of creep and relaxation can be written using fraction-exponential kernel (5) as

$$
\left(\mu^{*}\right)[\varepsilon(x, t)]=\mu_{0}\left[\varepsilon(x, t)+\lambda \int_{0}^{t} \ni_{\alpha}\left(\beta, t-t^{\prime}\right) \varepsilon\left(x, t^{\prime}\right) d t^{\prime}\right]
$$




$$
\left(\mu^{*}\right)^{-1}[\sigma(x, t)]=\frac{1}{\mu_{0}}\left[\sigma(x, t)-\lambda \int_{0}^{t} \ni_{\alpha}\left(\beta-\lambda, t-t^{\prime}\right) \sigma\left(x, t^{\prime}\right) d t^{\prime}\right]
$$

These formulas clarify the physical meaning of parameter $\beta$ - it is inverse of the relaxation time $\tau$ in power $1+\alpha$ taken with negative sign:

$$
\beta=\frac{1}{\tau^{1+\alpha}}
$$

It is also seen from (9) and (8) that

$$
\lambda=\frac{\mu_{0}-\mu_{\infty}}{\mu_{0}} \beta
$$

where $\mu_{\infty}$ is the shear modulus at $t \rightarrow \infty$ and $\mu_{0}$ is instantaneous shear modulus. Since in the processes of creep and relaxation shear modulus is decreasing function of time $\left(\mu_{0} \geq \mu_{\infty}\right)$, we have

$$
\beta<\lambda<0
$$

Thus, viscoelastic behaviour of a material is described by four parameters: $\mu_{0}, \alpha, \beta$ (or $\tau$ ), and $\lambda$ (or $\mu_{\infty}$ ). Connection between fraction-exponential operator was discussed by Rabotnov (1977). When $\alpha=0$ in (4) - (10), one can write

$$
(\mu)^{*}=\mu_{0}\left(1+\lambda \ni_{0}^{*}(\beta)\right)
$$

where the kernel of the operator is reduced to the ordinary exponential function $e^{-\beta t}$. In this case it describes the properties of the "standard" viscoelastic material represented by a combination of two springs with stiffness $E_{1}$ and $E_{2}$ and a dashpot of viscosity $\eta$ :

$\left(\frac{1}{E_{1}}+\frac{1}{E_{2}}\right) \sigma+\frac{\eta}{E_{1} E_{2}} \frac{d \sigma}{d t}=\varepsilon+\frac{\eta}{E_{2}} \frac{d \varepsilon}{d t}$.

The parameters of this model are expressed via $\mu_{0}, \lambda$ and $\beta$ as follows

$$
E_{1}=\mu_{0}, \quad E_{2}=\mu_{0}\left(\frac{\beta}{\lambda}-1\right), \quad \eta=\frac{\mu_{0}}{\lambda} .
$$


The Laplace transform of the fraction-exponential kernel has the following form:

$$
\mathcal{L}\left[\ni_{\alpha}^{*}(\beta, t)\right] \equiv \int_{0}^{\infty} \ni_{\alpha}^{*}(\beta, t) e^{-t p} d t=\frac{1}{p^{1-\alpha}+\beta}
$$

Thus, if elastic solution can be represented as a rational function of parameter $x=p^{1-\alpha}$, its inverse Laplace transform can be obtained analytically in an explicit form.

\section{Material and method}

\subsection{Synthesis of bacterial cellulose hydrogel}

Gluconacetobacter xylinum (ATCC53582) was used for bio-synthesis of the studied BC hydrogel. The bacterium was cultured in a Hestrin and Schramm (HS) medium, which was composed of 2 wt.\% glucose, 0.5 wt.\% yeast extract, 0.5 wt.\% peptone, 0.27 wt.\% disodium phosphate, and 0.15 wt.\% citric acid. After incubating statically for 7 days at $30^{\circ} \mathrm{C}$, resulting in thickness of the BC hydrogel in the range approximately from $3 \mathrm{~mm}$ to $5 \mathrm{~mm}$, the BC hydrogel was dipped into deionized (DI) water for 2 days, and then steamed by boiling in a $1 \mathrm{wt} . \% \mathrm{NaOH}$ solution for 30 mins to eliminate bacteria and proteins. Afterwards, the samples of BC hydrogels (FIG.1a) were purified by washing in DI water until their $\mathrm{pH}$ value approached 7 , and then, were stored in DI water at $4^{\circ} \mathrm{C}$.

\subsection{Creep tests}

Dog-bone-shape specimens for creep tests were cut with a custom-made die based on dimensions from ASTM-Standard 638-10 (FIG. 2a). Then, they were subjected to in-aqua tensile creep in a force-controlled mode employing a universal testing machine (Instron 3366, Instron, USA). A BioBuls system (Instron 3130-100 BioPuls Bath, Instron, USA) provided aqueous testing environment with constant temperature of $37.0 \pm 1.0^{\circ} \mathrm{C}$ (FIG. 2b). A pre-loading of $0.05 \mathrm{~N}$ was set in tests. Specimens were first stretched to $50 \%, 60 \%, 70 \%$ and $80 \%$ of ultimate stress (1.43 $\mathrm{MPa}$ ), and then stress was hold for 1.5 hrs. A $100 \mathrm{~N}$ load cell (2530 Series Low-profile Static Load Cell, Instron, USA) and a crosshead displacement sensor were used to measure the force and displacement, respectively, with data acquisition frequency of $1 \mathrm{~Hz}$.

\subsection{Assessment of fraction-exponential parameters}

A dedicated numerical procedure to fit the experimental data using a fraction-exponential approach was developed. The implementation of Mittag-Leffler function $M$ available at MATLAB CENTRAL was used to approximate a stress-strain relation (obtained as a combination of equations (6) and (9)): 
$\varepsilon(t)=\frac{\sigma_{0}}{\mu_{0}}\left\{1-\frac{\lambda}{\beta-\lambda}\left[\mathrm{M}_{\alpha+1}\left((\beta-\lambda) t^{\alpha+1}\right)-1\right]\right\}$

The magnitude of $\mu_{0}$ was found as $\mu_{0}=\frac{1}{\varepsilon(0)}$. Values of the remaining parameters $\alpha, \beta$, and $\lambda$ - were approximated with a nonlinear least-square method. In our case, this was performed using a MATLAB lsqnonlin function with initial guesses of $-0.5,-0.1$, and 0.0005 respectively. The accuracy of the approximation was calculated with Resnorm $R$ that is $l^{2}$-norm of fit to present the accuracy of curve fitting, defining as,

$$
R=\sum\left[\varepsilon_{E}(t)-\varepsilon_{F}(t)\right]^{2}
$$

where $\varepsilon_{E}(t)$ is the experimental strain data, $\varepsilon_{F}(t)$ is the derived function. The desired accuracy of this approximation was set to be 0.0001 .

\section{Results and Discussion}

The experimentally determined creep (strain-time) curves at stress levels of 50\%, 60\%, 70\% and $80 \%$ of ultimate strength $\sigma_{U L T}$ are shown in FIG. 3. The fraction-exponential model with four

material parameters was developed only to describe the time-dependent behaviour; thus, the plots presented in FIG. 3 omit the pre-creep domain. A non-linear time-dependent behaviour was observed for all the applied stress levels. Specimens demonstrated typical creep behaviour: after some $1000 \mathrm{~s}$, a secondary-creep period was initiated. At the studied highest stress level (80\% of ), at around $4500 \mathrm{~s}$ corresponding to the strain magnitude of around $70.5 \%$, an increase in the creep strain started to accelerate, manifesting a transition to a tertiary creep. That is caused by significant microstructural changes accumulated during the deformation process to such advanced levels of stretching that might be accompanied by failure of some nanofibres, leading to local stress concentration.

On the basis of physical meanings of parameters used in the fraction-exponential formalism, the developed numerical fitting procedure provided the best fit for the experimental data employing terms of fraction-exponential operators. A comparison of the obtained experimental results with fraction-exponential approximations with various sets of parameters for each stress level is shown in FIG. 4. The determined parameters with respective levels of Resnorm for each stress level are summarized in TABLE 1 . Apparently, the determined parameters meet the basic requirements of the fraction-exponential operators $(-1<\alpha \leq 0, \beta<\lambda<0)$. Resnorm (fit accuracy) was calculated to be less than 0.0001 . As a result, the fitting curves show an excellent agreement with experimental data for both primary and secondary creep, demonstrating that the fraction-exponential model is suitable to describe the time-dependent rheological behaviour of BC hydrogel. 
Obviously, it is desirable to have constant values of fraction-exponential parameters for a specific material, independent of the load magnitude, but the studied BC hydrogel demonstrated stress-sensitivity of them. Since the obtained parameters in TABLE 1 do not show large difference, at the next stage of the analysis a single set of parameters, providing a. Then, we a best fit for experimental data for all the stress levels, was determined using the developed MATLAB procedure. This single set is presented in TABLE 2 alongside the Resnorm magnitudes for the studied stresses; the respective plots are shown in FIG. 5. The error level of the theoretical approximation is around $10 \%$, not suitable for many practical cases. A comparison of analytical and experimental curves in FIG. 5 suggests that these differences are mainly due to separation between the initial points of these two types of curves.

As shown in FIG. 6, due to fibre engagement in a load-bearing process with growing stretching, the microstructure of BC hydrogel changes with increasing stress level. This includes changes in orientation distribution of fibres, their local volume fraction, porosity and interaction between fibres and water (Astley et al., 2003; Frensemeier et al., 2010; Miao and Hamad, 2013), resulting in a material stiffening process. Hence, the tensile creep behaviour of the BC hydrogel should vary with the increased stress level. In the fraction-exponential model, the parameter of $\mu_{0}$ represents the instantaneous shear modus, positively correlating to the stiffness of BC specimens. Thereby, it substantially increases with increasing stress. In the numerical fitting procedure, $\mu_{0}$ was determined at the initial point of the experimental creep data $(t=0)$. It increased with the increased stress levels (TABLE 1), coinciding with a notion of its physical meaning - material stiffening. So, at the next stage of the analysis, the same values of $\mu_{0}$ in TABLE 1 were used to define a single set of other three parameters of the fraction-exponential formalism: $\lambda, \alpha$ and $\beta$ with a best fit for all stress levels, as shown in FIG. 7. The determined parameters are summarized in TABLE 3 together with respective Resnorm Values. Apparently, the latter is less than $10^{-2}$, demonstrating sufficient accuracy of the developed fraction-exponential model in description of the creep behaviour of BC hydrogel. It is interesting to note that in such an approximation, the dependence of the instantaneous shear modulus $\mu_{0}$ on the level of creep stress is practically linear (FIG. 8).

\section{Conclusions}

The time-dependent rheological behaviour of the bacterial cellulose hydrogel is assessed with the uniaxial tensile creep tests performed at various stress levels, up to $80 \% \sigma_{U L T}$. The experimental analysis was accompanied by an analytical study employing a fraction-exponential formalism for rheological behaviour used to model the creep data for the studied BC hydrogel. Such formalism is especially important for development of analytical tools (including numerical, e.g. finite-element schemes) for solving boundary value problems for viscoelastic materials, arising in biomedical applications. Both the primary and the secondary creep are described with sufficient accuracy at stress levels ranging from $50 \%$ to $80 \% \sigma_{U L T}$, relevant for many practical 
cases. The physical meaning of the determined material parameters involved in the model is discussed. The developed numerical procedure allows definition of the magnitudes of fraction-exponential parameters. When applied to creep at each stress level, it provides a highly accurate approximation of experimental data. A more suitable for numerical analysis approaches can be based on a single set of three parameters together with an instantaneous shear modulus, changing linearly with the level of creep stress. The latter reflects a physical mechanism of material stiffening caused by fibre reorientation under axial stretching.

\section{Acknowledgement}

The partial support from the following grants is gratefully acknowledged: FP7 IRSES project TAMER (Grant No. IRSES-GA-2013-610547) (XG, PK, IS and VVS); China-European Union technology cooperation programme (Grant No. 1110) (ZS and GY); M6 Project (Grant No. PIRSES-GA-2010-269113) (CL and VVS).

\section{References}

Astley, O.M., Chanliaud, E., Donald, A.M., and Gidley, M.J., "Tensile deformation of bacterial cellulose composites," Int. J. Biol. Macromol. 32, 28-35 (2003).

Bäckdahl, H., Esguerra, M., Delbro, D., Risberg, B., and Gatenholm, P., "Engineering microporosity in bacterial cellulose scaffolds," J. Tissue Eng. Regen. Med. 2, 320-330 (2008).

Bäckdahl, H., Helenius, G., Bodin, A., Nannmark, U., Johansson, B. R., Risberg, B., and Gatenholm, P., "Mechanical properties of bacterial cellulose and interactions with smooth muscle cells," Biomaterials 27, 2141-2149 (2006).

Brown, E.E., Laborie, M.G., and Zhang, J., "Glutaraldehyde treatment of bacterial cellulose/fibrin composites: impact on morphology, tensile and viscoelastic properties," Cellulose 19, 127-137 (2012).

Brown, E.E., Zhang, J., and Laborie, M.G., "Never-dried bacterial cellulose/fibrin composites: preparation, morphology and mechanical properties," Cellulose 18, 631-641 (2011).

Christensen, R.M., Theory of Viscoelasticity, Academic Press, (1982).

Di Paola, M., and Zingales, M., "Exact mechanical models of fractional hereditary materials," J. Rheol. 56, 983-1004 (2012).

Frensemeier, M., Koplin, C., Jaeger, R., Kramer, F., and Klemm, D., "Mechanical Properties of Bacterially Synthesized Nanocellulose Hydrogels," Macromol. Symp. 294, 38-44 (2010).

Gibas, I., and Janik, H., "Review: synthetic polymer hydrogels for biomedical applications," Ch\&ChT. 4, 297-304 (2010).

Guhados, G., Wan, W., and Hutter. J.L., "Measurement of the Elastic Modulus of Single Bacterial Cellulose Fibers Using Atomic Force Microscopy," Langmuir 21, 6642-6646 (2005).

Kim, J., Ampofo, J., Craft, W., and Kim, H.S., "Modeling elastic, viscous, and creep characteristics of cellulose Electro-Active Paper," Mech. Mater. 40, 1001-1011 (2008). 
Klemm, D., Schumann, D., Kramer, F., Heßler, N., Hornung, M., Schmauder, H.-P., and Marsch, S., "Nanocelluloses as Innovative Polymers in Research and Application," Adv. Polym. Sci. 205, 49-96 (2006).

Kowalska-Ludwicka, K., Cala, J., Grobelski, B., Sygut, D., Jesionek-Kupnicka, D., Kolodziejczyk, M., Bielecki, S., and Pasieka, Z., "Modified bacterial cellulose tubes for regeneration of damaged peripheral nerves," Arch. Med. Sci. 9, 527-534 (2013).

Levin, V., and Sevostianov, I., "Micromechanical modeling of the effective viscoelastic properties of inhomogeneous materials using fraction-exponential operators," Int. J. Fracture 134, L37-L44 (2005).

Lopez-Sanchez, P., Rincon, M., Wang, D., Brulhart, S., Stokes, J.R., and Gidley, M.J., "Micromechanics and Poroelasticity of Hydrated Cellulose Networks," Biomacromolecules 15, 2274-2284 (2014).

Malm, C. J., Risberg, B., Bodin, A., Bäckdahl, H., Johansson, B. R., Gatenholm, P., and Jeppsson, A., "Small calibre biosynthetic bacterial cellulose blood vessels: 13-months patency in a sheep model," Scand. Cardiovasc. J. 46, 57-62 (2012).

Miao, C., and Hamad, W.Y., "Cellulose reinforced polymer composites and nanocomposites: a critical review," Cellulose 20, 2221-2262 (2013).

Nimeskern, L., Martínez Ávila, H., Sundberg, J., Gatenholm, P., Müller, R., and Stok, K. S., "Mechanical evaluation of bacterial nanocellulose as an implant material for ear cartilage replacement," J. Mech. Behav. Biomed. Mater. 22, 12-21 (2013).

Podlubny, I., Fractional Differential Equations, Academic Press (1998).

Rabotnov, Yu.N., "Equilibrium of an elastic medium with after-effects. (in Russian)," J. Appl. Math. Mech. 12, 53-62 (1948).

Rabotnov, Yu.N., Elements of Hereditary Solid Mechanics, Moscow: Mir (1977).

Scott Blair, G.W., and Coppen, F.M.V., "The subjective judgement of the elastic and plastic properties of soft bodies: the differential thresholds for viscosities and compression moduli," Proc. Roy. Soc. B 128, 109-125 (1939).

Scott Blair, G.W., and Coppen, F.M.V., "The estimation of firmness in soft materials," Amer. J. Psychol. 56, 234-246 (1943).

Sevostianov, I., and Levin, V., "Creep and relaxation contribution tensors for spheroidal pores in hereditary solids: fraction-exponential operators approach," Acta Mech., (in press), (2015).

Sevostianov, I., Levin, V.M., and Radi, E., "Effective properties of linear viscoelastic microcracked materials: application of Maxwell homogenization scheme," Mech. Mater. (in press), (2015).

Shi, Z., Zhang, Y., Phillips, G. O., and Yang, G., "Utilization of bacterial cellulose in food," Food Hydrocolloids 35, 539-545 (2014).

Wang, J., Gao, C., Zhang, Y. and Wan, Y., "Preparation and in vitro characterization of BC/PVA hydrogel composite for its potential use as artificial cornea biomaterial," Mater. Sci. Eng. C 30, 214-218 (2010). 
Zang, S., Zhang, R., Chen, H., Lu, Y., Zhou, J., Chang X., Qiu, G., Wu, Z. and Yang, G., "Investigation on artificial blood vessels prepared from bacterial cellulose," Mater. Sci. Eng. C 46, 111-117 (2015). 


\section{Figures}
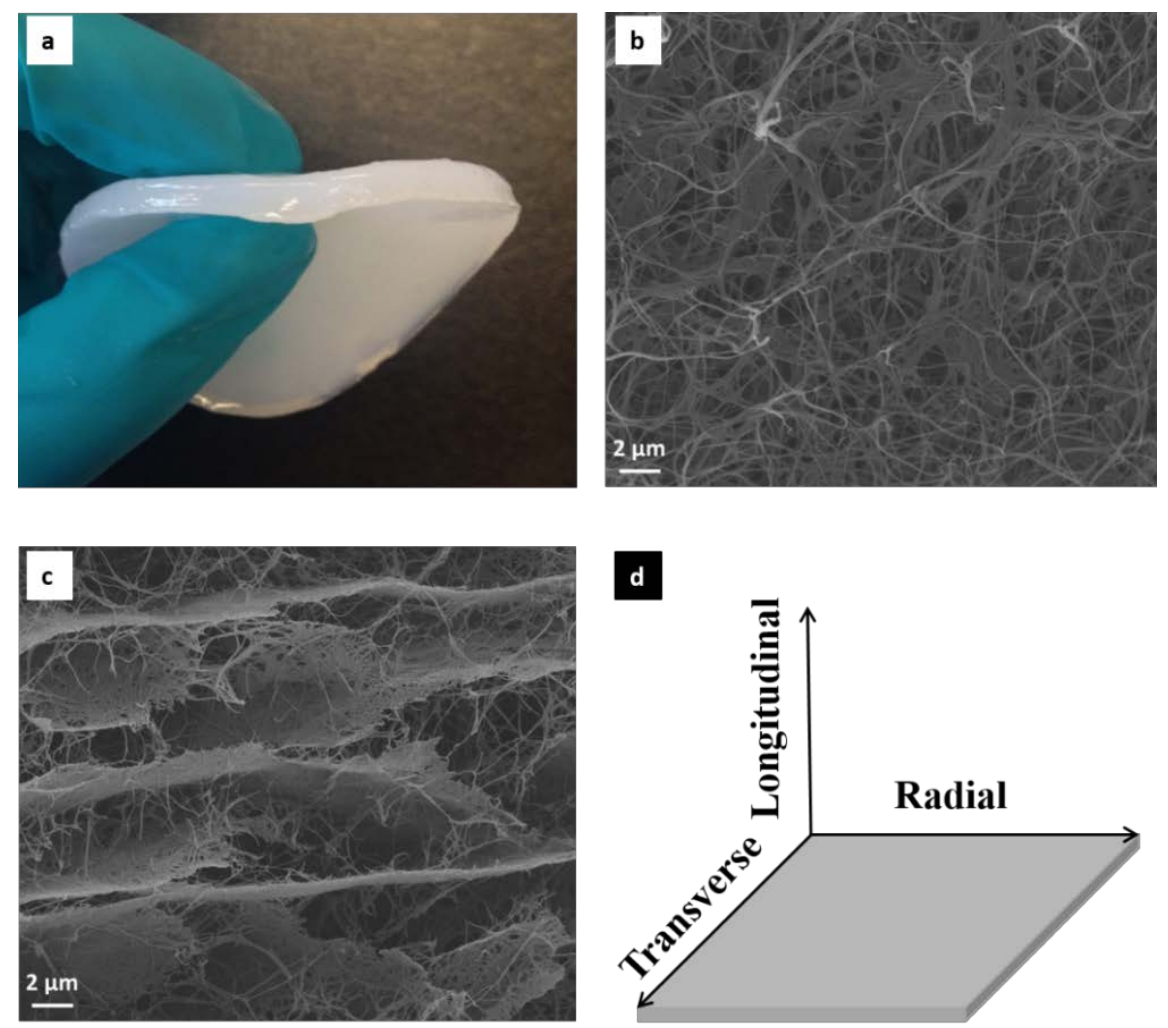

FIG. 1: (a) Disk sample of wet BC hydrogel's. SEM images of BC hydrogel shows a random distribution of cellulose fibres in the radial-transverse plane (b) and multiple-layer structure with some links between layers along out-of-plane direction(c). (d) Schematic diagram of radial, transverse and out-of-plane directions.
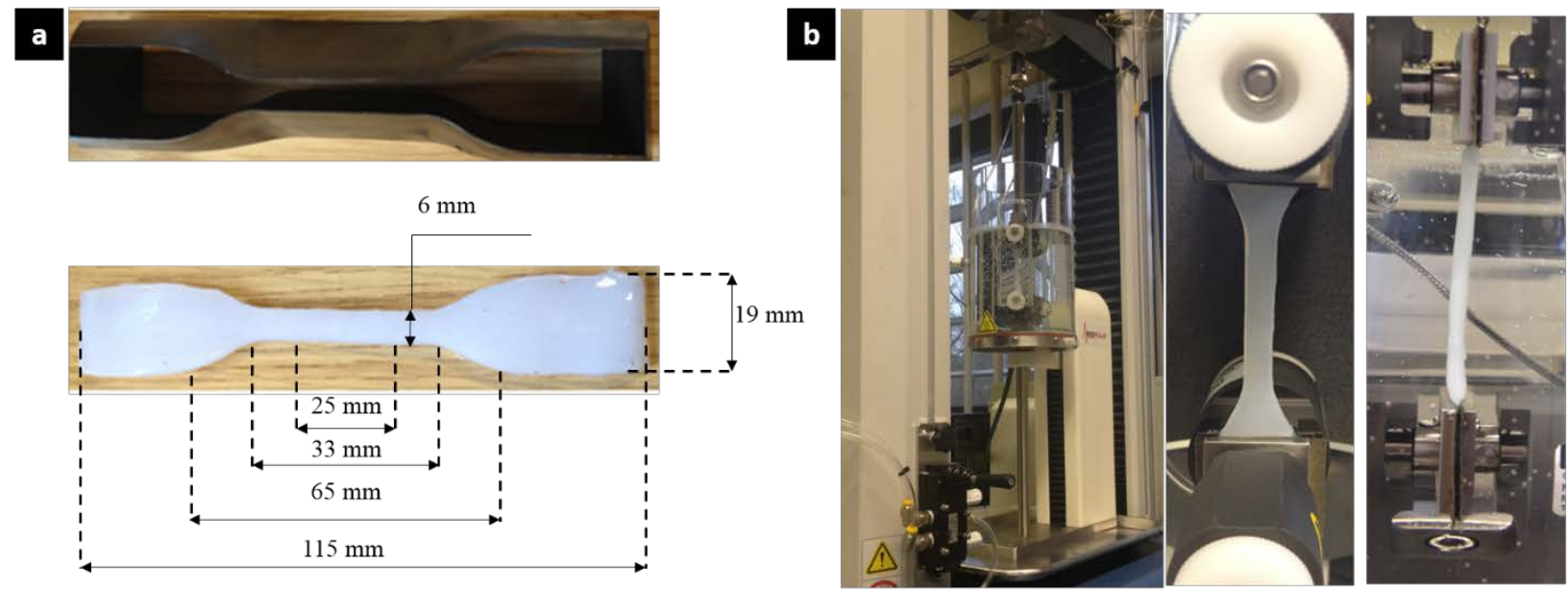

FIG. 2: (a) Dog-bone-shape specimen with dimensions suggested by ASTM 638-10 standard for tensile creep tests cut with a custom-made die. (b) Instron BioPuls system providing aqueous testing condition at constant temperature of $37.0 \pm 1.0^{\circ} \mathrm{C}$ 

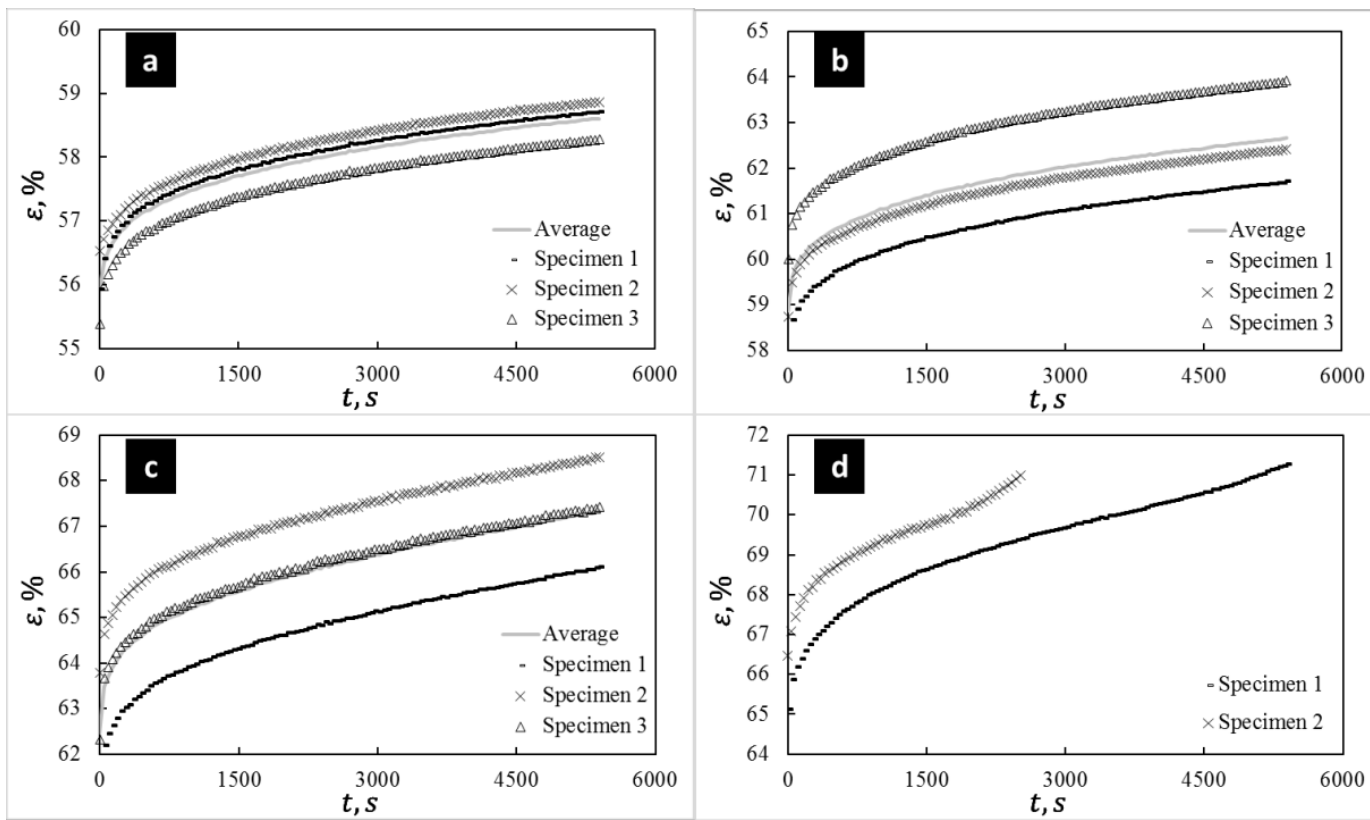

FIG. 3: Evolution of strain with increased time shows typical creep behaviour of BC hydrogel (3 specimens for each level of stresses) at various stress levels: (a) $50 \% \sigma_{U L T}$; (b) $60 \% \sigma_{U L T}$; (c) $70 \% \sigma_{U L T} ;$ (d) $80 \% \sigma_{U L T}$.
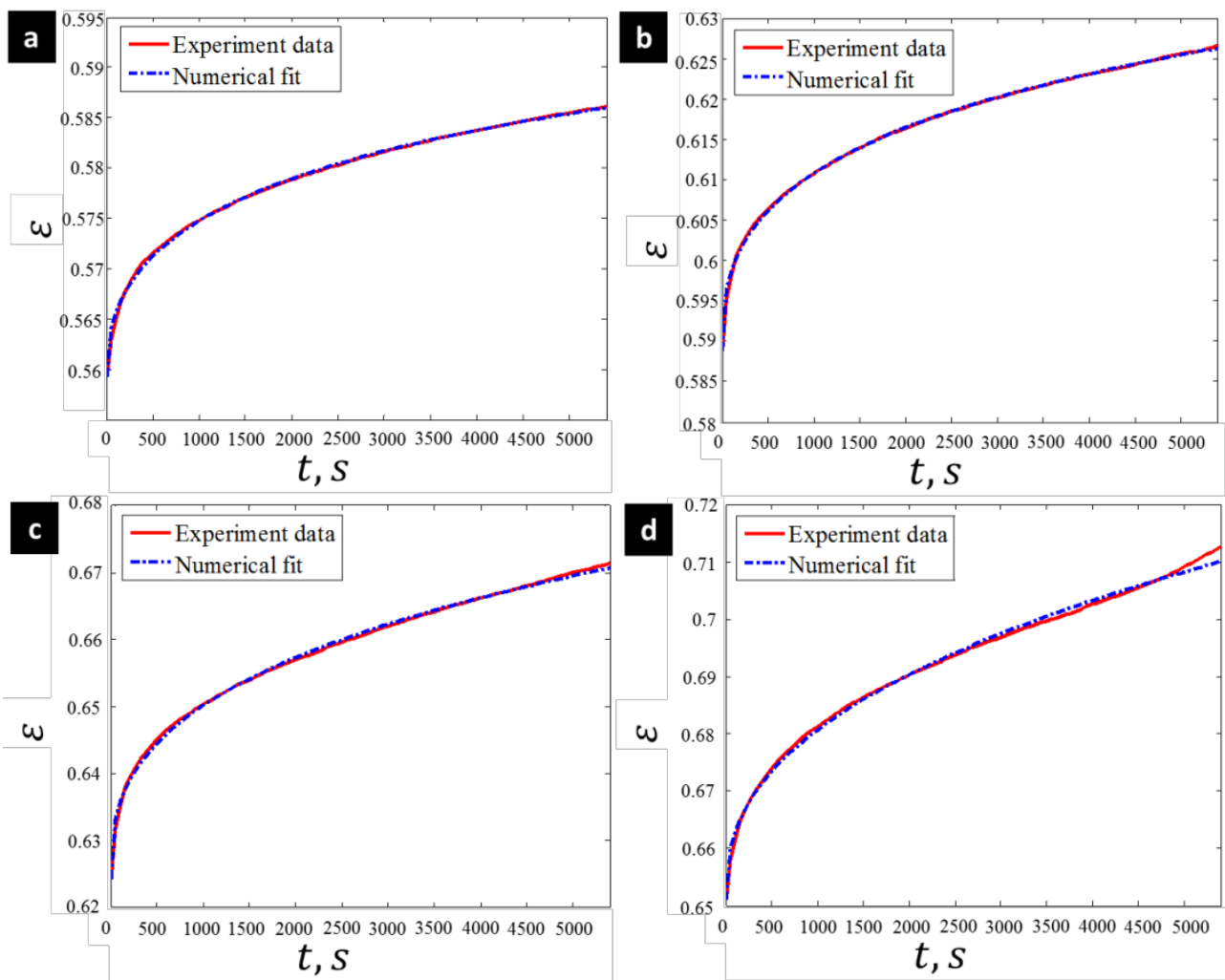

FIG. 4: The fraction-exponential model is sensitive enough to describe the creep behaviour of $\mathrm{BC}$ hydrogel. The numerical fit curves with various parameters show perfect agreement with experimental ones at various stress levels: (a) $50 \% \sigma_{U L T}$; (b) $60 \% \sigma_{U L T}$; (c) $70 \% \sigma_{U L T}$; (d) $80 \%$ $\sigma_{U L T}$. 

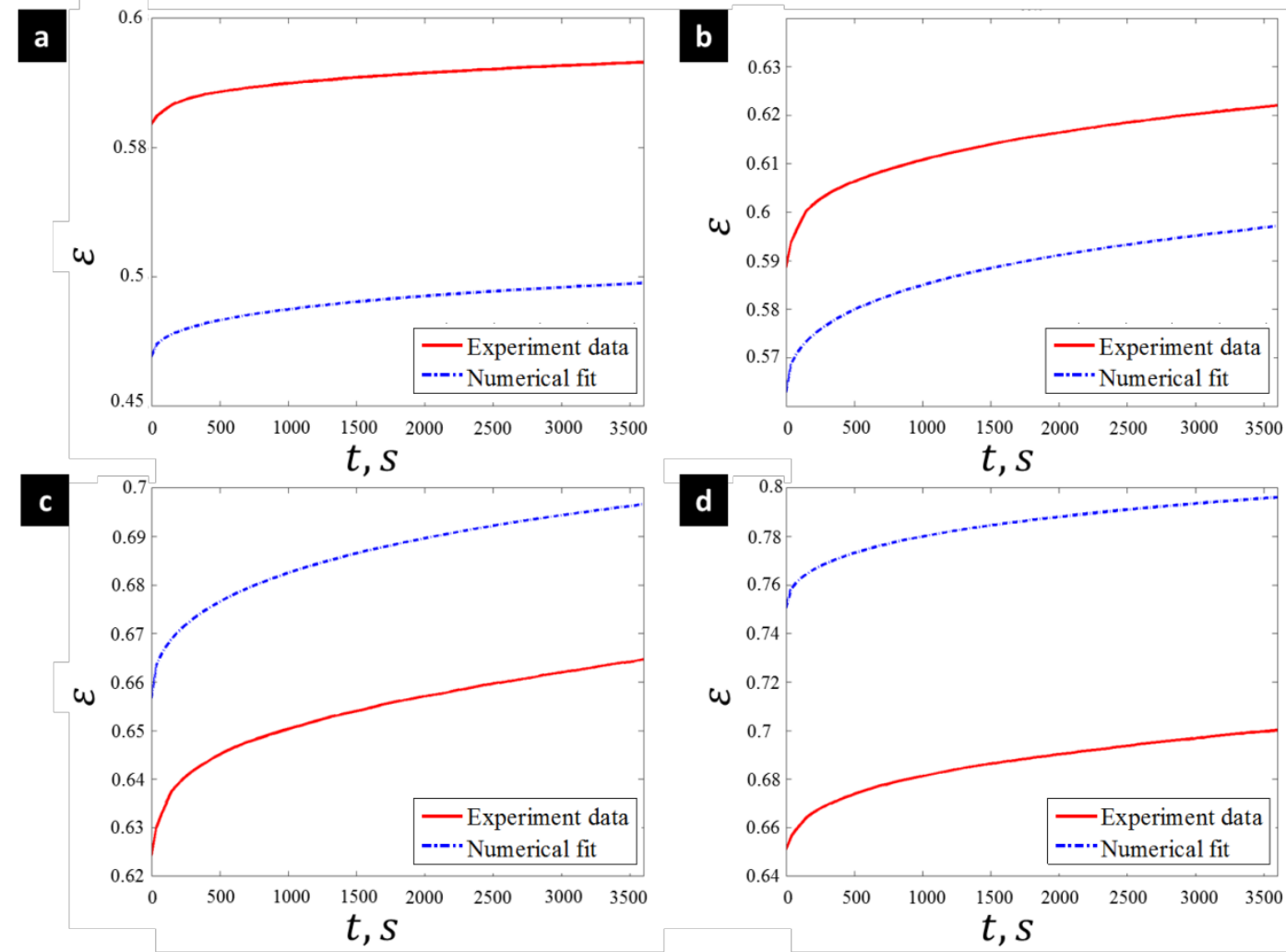

FIG. 5: The fraction-exponential model with constant parameters shows large errors at stress levels of $50 \% \sigma_{U L T}$ (a), 60\% $\sigma_{U L T}$ (b), 70\% $\sigma_{U L T}$ (c) and $80 \% \sigma_{U L T}$ (d), mainly due to the separation between the initial points of the experimental and numerical curves.

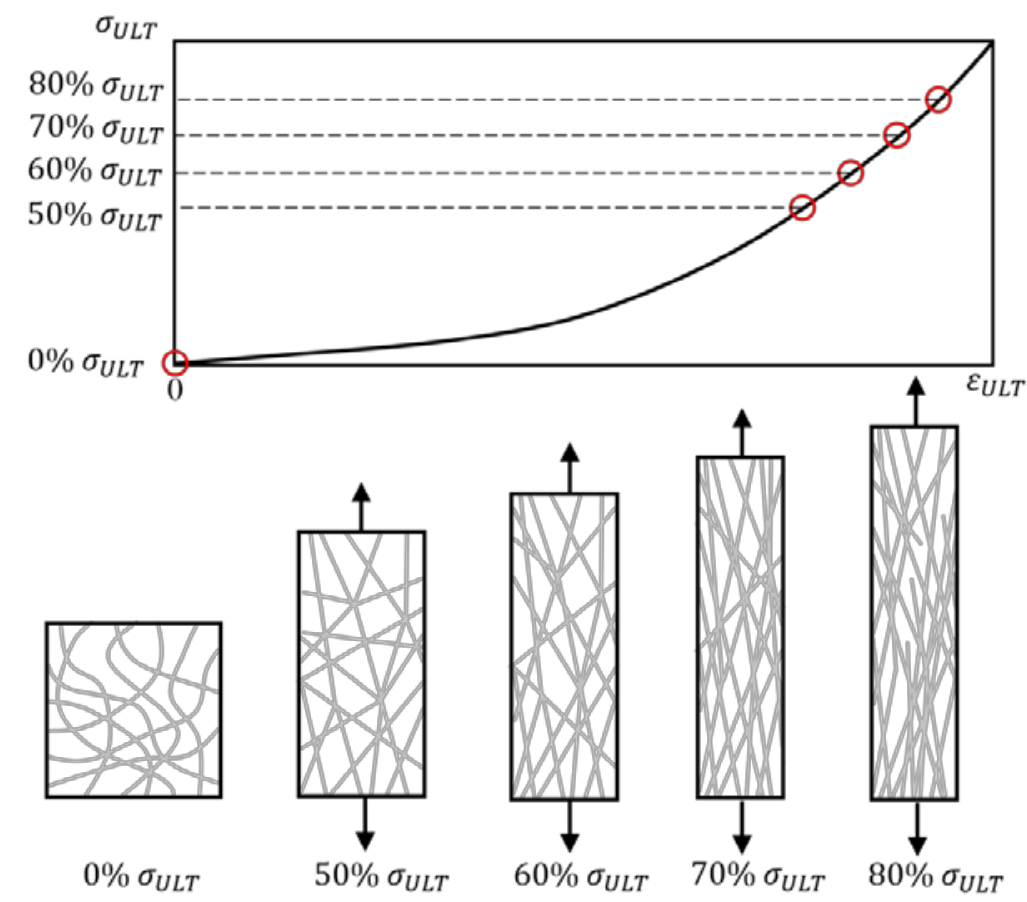

FIG. 6: A schematic diagram of microstructural change at increased stress levels ranging from $50 \%$ $\sigma_{U L T}$ to $80 \% \sigma_{U L T}$, indicating a process of fibre rearrangement resulting in material stiffening. 

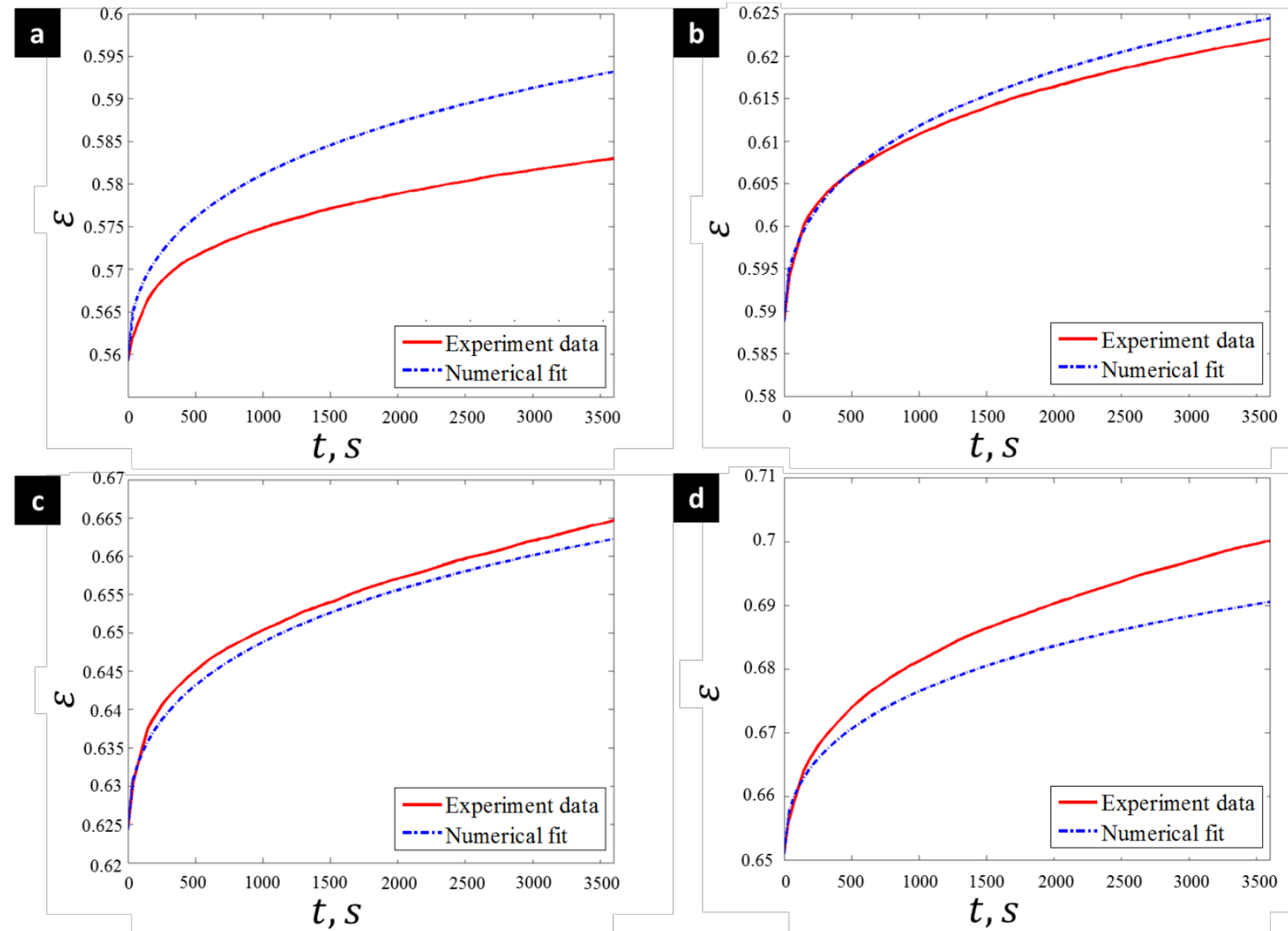

FIG. 7: The fraction-exponential model with various $\mu_{0}$ and constant $\lambda, \alpha$ and $\beta$ for stress levels shows sufficient accuracy with the experimental data for stress levels of $50 \% \sigma_{U L T}$ (a), 60\% $\sigma_{U L T}$ (b), $70 \% \sigma_{U L T}$ (c) and $80 \% \sigma_{U L T}$ (d), indicating the possibility to use the developed model for description of creep behaviour of the studied BC hydrogel.

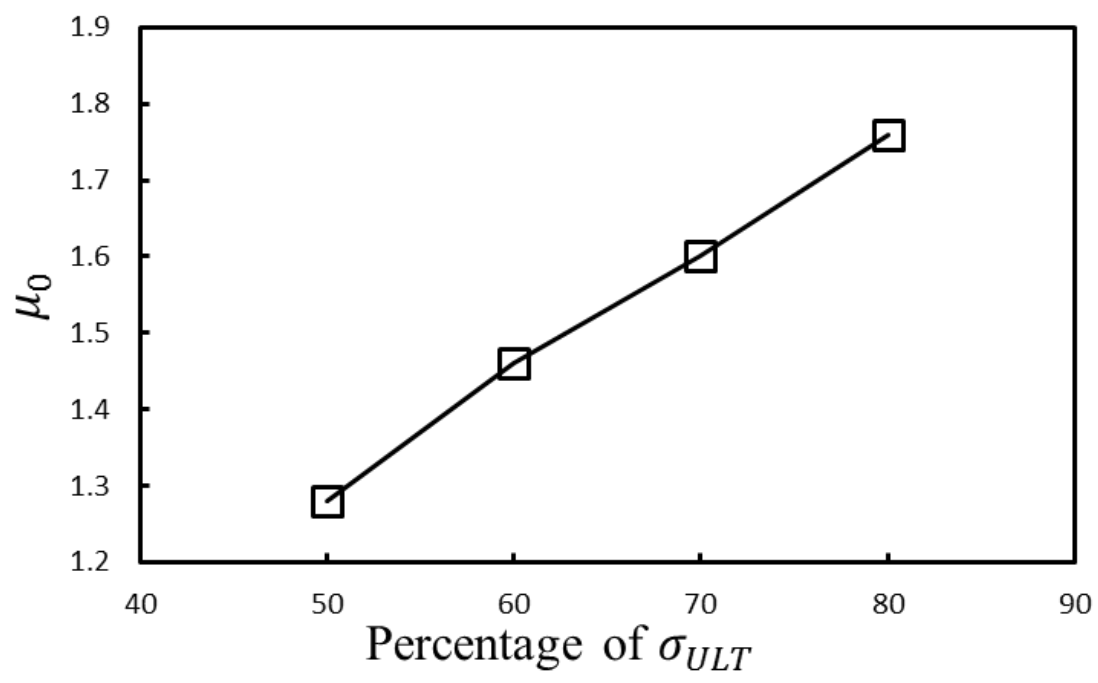

FIG. 8: Evolution of instantaneous shear modulus $\mu_{0}$ with stress level, indicating material stiffening coinciding with fibre-rearrangement theory. 
Table

TABLE 1: Determined fraction-exponential parameters of various $\mu_{0}, \lambda, \alpha$ and $\beta$, and Resnorm $\mathrm{R}$ for each stress levels

\begin{tabular}{cccccc}
\hline $\begin{array}{c}\text { Proportion of } \\
\text { ultimate stress }\end{array}$ & $\boldsymbol{\mu}_{\mathbf{0}}$ & $\boldsymbol{\lambda}$ & $\boldsymbol{\alpha}$ & $\boldsymbol{\beta}$ & $\begin{array}{c}\mathbf{R} \\
\text { (fit accuracy) }\end{array}$ \\
\hline $\mathbf{5 0 \%}$ & 1.28 & $-1.34 \times 10^{-3}$ & -0.55 & $-1.31 \times 10^{-2}$ & $3.18 \times 10^{-6}$ \\
$\mathbf{6 0 \%}$ & 1.46 & $-2.50 \times 10^{-3}$ & -0.60 & $-1.43 \times 10^{-2}$ & $3.53 \times 10^{-6}$ \\
$\mathbf{7 0} \%$ & 1.60 & $-2.79 \times 10^{-3}$ & -0.61 & $-8.12 \times 10^{-3}$ & $1.42 \times 10^{-5}$ \\
$\mathbf{8 0 \%}$ & 1.76 & $-1.96 \times 10^{-3}$ & -0.54 & $-7.58 \times 10^{-3}$ & $1.48 \times 10^{-5}$ \\
\hline
\end{tabular}

TABLE 2: Determined fraction-exponential parameters of constant $\mu_{0}, \lambda, \alpha$ and $\beta$, and Resnorm $\mathrm{R}$ for each stress levels

\begin{tabular}{cccccc}
\hline $\begin{array}{c}\text { Proportion of } \\
\text { ultimate stress }\end{array}$ & $\boldsymbol{\mu}_{\mathbf{0}}$ & $\boldsymbol{\lambda}$ & $\boldsymbol{\alpha}$ & $\boldsymbol{\beta}$ & $\begin{array}{c}\mathbf{R} \\
\text { (fit accuracy) }\end{array}$ \\
\hline $\mathbf{5 0 \%}$ & & & & & $7.52 \times 10^{-1}$ \\
$\mathbf{6 0 \%}$ & \multirow{2}{*}{$* 52$} & $-1.85 \times 10^{-3}$ & -0.55 & $-1.24 \times 10^{-2}$ & $6.53 \times 10^{-2}$ \\
$\mathbf{7 0 \%}$ & & & & & $1.04 \times 10^{-1}$ \\
$\mathbf{8 0 \%}$ & & & & & $9.61 \times 10^{-1}$ \\
\hline
\end{tabular}

TABLE 3: Determined fraction-exponential parameters of various $\mu_{0}$, and constant $\lambda$, $\alpha$ and $\beta$, and Resnorm $\mathrm{R}$ for each stress levels

\begin{tabular}{cccccc}
\hline $\begin{array}{c}\text { Proportion of } \\
\text { ultimate stress }\end{array}$ & $\boldsymbol{\mu}_{\mathbf{0}}$ & $\boldsymbol{\lambda}$ & $\boldsymbol{\alpha}$ & $\boldsymbol{\beta}$ & $\begin{array}{c}\mathbf{R} \\
\text { (fit accuracy) }\end{array}$ \\
\hline $\mathbf{5 0 \%}$ & 1.28 & & & & $6.03 \times 10^{-3}$ \\
$\mathbf{6 0 \%}$ & 1.46 & & & & $2.65 \times 10^{-4}$ \\
$\mathbf{7 0 \%}$ & 1.60 & $-1.85 \times 10^{-3}$ & -0.55 & $-1.24 \times 10^{-2}$ & $2.92 \times 10^{-4}$ \\
$\mathbf{8 0 \%}$ & 1.76 & & & & $4.27 \times 10^{-3}$ \\
\hline
\end{tabular}

